(C) [2009] IEEE. Reprinted, with permission, from Xu, Wei; Zhu, Jianguo; Tan, Longcheng; Guo, Youguang; Wang, Shuhong; Wang, Yi. 2009, 'Optimal Design of a Linear Induction Motor Applied in Transportation', Proceedings of International Conference on Industrial Technology, pp. 790-795.This material is posted here with permission of the IEEE. Such permission of the IEEE does not in any way imply IEEE endorsement of any of the University of Technology, Sydney's products or services. Internal or personal use of this material is permitted. However, permission to reprint/republish this material for advertising or promotional purposes or for creating new collective works for resale or redistribution must be obtained from the IEEE by writing to pubs-permissions@ieee.org. By choosing to view this document, you agree to all provisions of the copyright laws protecting it. 


\title{
Optimal Design of a Linear Induction Motor Applied in Transportation
}

\author{
Wei $\mathrm{Xu}^{1}$, Jianguo Zhu ${ }^{1}$, Longcheng $\mathrm{Tan}^{2}$, Youguang Guo ${ }^{1}$, Shuhong Wang ${ }^{1,3}$, and Yi Wang ${ }^{1}$ \\ (1. School of Electrical, Mechanical and Mechatronic Systems, University of Technology, Sydney, NSW 2007, Australia \\ 2. Institute of Electrical Engineering, Chinese Academy of Sciences, Beijing 100190, China \\ 3. Faculty of Electrical Engineering, Xi'an Jiaotong University, Xi'an 710049, China) \\ E-mail: wxu@eng.uts.edu.au
}

\begin{abstract}
Several optimal design schemes of a single-sided linear induction motor (SLIM) adopted in linear metro are presented in this paper. Firstly, the equivalent circuit of SLIM fully considering the end effects, half-filled slots, back-iron saturation and skin effect is proposed, based on one-dimensional air-gap magnetic equations. In the circuit, several coefficients including longitudinal end effect coefficients $K_{\mathrm{r}}(\mathrm{s})$ and $K_{\mathrm{x}}(\mathrm{s})$, transversal end edge effect coefficients $C_{\mathrm{r}}(\mathrm{s})$ and $C_{\mathrm{x}}(\mathrm{s})$, and skin effect coefficient $K_{\mathrm{f}}$ are achieved by using the dummy electric potential method and complex power equivalence between primary and secondary sides. Furthermore, several optimal design restraint equations of SLIM are provided in order to improve the operational efficiency and reduce the primary weight. These nonlinear equations are solved by using genetic algorithm and mixture penalty function method. The optimal schemes are compared with the original design of one company, where analysis on parameters is made in detail. These results show that the optimal schemes are reasonable for improving the performance of SLIM.
\end{abstract}

\section{INTRODUCTION}

In the recent years, the single-sided linear induction motor (SLIM) has been widely adopted in transportation systems, especially in intermediate speed range. Typical examples include the HSST in Japan and the linear metro in Canada, where power systems are both propelled by the SLIMs [1-3].

The SLIM has the following merits comparing with the rotary induction motor (RIM): greater ability to exert thrust on the secondary without mechanical contacts, higher acceleration or deceleration, less wear of the wheels, smaller turn circle radius, and more flexible road line.

Because of its cut-open magnetic circuit, the linear induction motor (LIM) possesses the inherent characteristics such as longitudinal end-effect, transversal edge-effect and normal force. In addition, it also has half-filled slots in the primary ends. Therefore, an accurate equivalent circuit model of LIM is difficult to be obtained compared to that of RIM [4].

Many analysis techniques of SLIM have been studied and developed in the past years. However, effective methods on the design scheme of SLIM have not been obtained due to the following reasons. The selection of electric loading and magnetic loading by loading distribution is so difficult that one cannot calculate the apparent power (kVA) easily. The power factor and efficiency are affected by the end effect which is again affected by the design technique of SLIM.
The pole pitch can be varied structurally and the selection of frequency and rated slip is not easy with the decision on the rated velocity [5].

A new improved model is set out in this paper based on the air-gap flux density equation, which fully takes into account the end effects, half-filled slots, back-iron saturation and skin effect. Then, optimal design equations for achieving the maximum efficiency and minimum primary weight are built, which are nonlinearly constrained. It is necessary to choose a suitable solving method to obtain the independent variables, such as the rated slip, number of poles, pole pitch, stack height, ratio of slot width to slot pitch, secondary aluminum (copper?) thickness, tooth magnetic density, and secondary overhang [4-7].

\section{PhysiCAl STRUCTURE AND MATHEMATICAL ANALYTIC MODEL}

The structure of an SLIM and its equivalent circuit are shown separately in Fig. 1 and Fig. 2.

In the analytic circuit, $K_{f}$ is the skin effect correction coefficient, $K_{r}(s)$ and $K_{x}(s)$ are the longitudinal end-effect coefficients, and $C_{r}(s)$ and $C_{x}(s)$ are the transversal end-effect coefficients [8]. These correction coefficients are expressed below.

The skin effect correction coefficient is

$$
K_{f}=\frac{1+B_{1}^{2} \operatorname{sh}^{2}\left(2 K \delta_{e}^{\prime}\right)}{A_{1}\left[1+B_{1}^{2} s^{2}(2 K d)\right]}
$$

where $K$ is the function of slip and motor structure, $d$ is the thickness of secondary metal sheet, $\delta_{\mathrm{e}}^{\prime}$ is the equivalent magnetic air gap length, and $A_{1}$ and $B_{1}$ are coefficients which are affected by slip and motor structure.

$\delta_{e}{ }^{\prime}$ can be expressed as

$$
\delta_{e}^{\prime}=K_{c} K_{u} g
$$

where $K_{c}$ is the Carter coefficient and $K_{u}$ is the magnetic saturation coefficient.

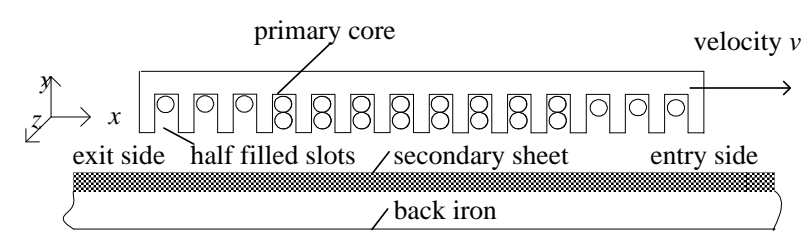


(a) Longitudinal side view

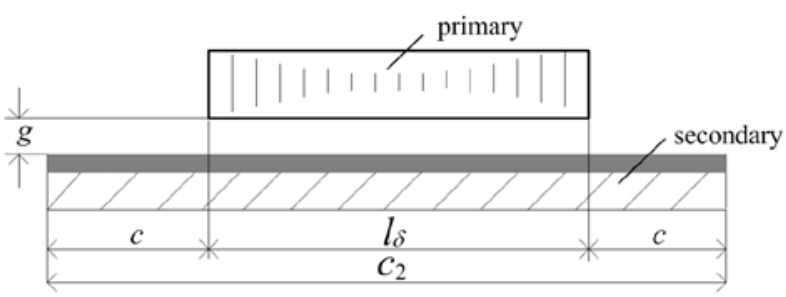

(b) Transversal side view

Fig. 1. Simple construction diagram of LIM

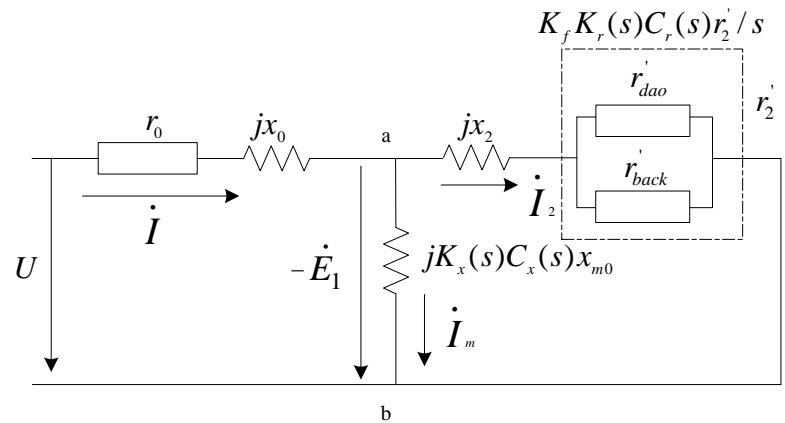

Fig. 2. T-type equivalent circuit of LIM considering the back iron resistance

$A_{1}$ and $B_{1}$ are derived as

$$
\begin{aligned}
& A_{1}=\operatorname{ch}^{2}\left(K \delta_{e}^{\prime}\right)+\left[\frac{K \rho_{\text {second }} \operatorname{sh}\left(K \delta_{e}^{\prime}\right)}{S \omega \mu_{0} d}\right]^{2} \\
& B_{1}=\frac{S \omega \mu_{0} d}{2 K \rho_{\text {second }}}\left[1+\frac{K \rho_{\text {second }}}{S \omega \mu_{0} d}\right]^{2}
\end{aligned}
$$

where $S$ is the slip, $\omega$ is the primary electrical angular velocity, $\rho_{\text {second }}$ is the resistivity of secondary electrical sheet, and $\mu_{0}$ is the permeability of air.

The longitudinal end-effect coefficients $K_{r}(s)$ and $K_{x}(s)$ are denoted by

$$
\begin{aligned}
& K_{r}(s)=\frac{S G}{2 P_{e} \tau \sqrt{1+(S G)^{2}}} \frac{C_{1}^{2}+C_{2}^{2}}{C_{1}} \\
& K_{x}(s)=\frac{1}{2 P_{e} \tau \sqrt{1+(S G)^{2}}} \frac{C_{1}^{2}+C_{2}^{2}}{C_{2}}
\end{aligned}
$$

where $C_{1}$ and $C_{2}$ are the functions of slip $S$ and goodness factor $G, P_{\mathrm{e}}$ is the number of equivalent pole pairs, and $\tau$ is the pole pitch.

The transversal end-effect coefficients $C_{r}(s)$ and $C_{x}(s)$ are given by

$$
\begin{aligned}
C_{r}(s) & =\frac{S G\left[\mathrm{R}_{e}^{2}[T]+\mathrm{I}_{m}^{2}[T]\right]}{\mathrm{R}_{\mathrm{e}}[T]} \\
C_{x}(s) & =\frac{\mathrm{R}_{e}^{2}[T]+\mathrm{I}_{m}^{2}[T]}{\mathrm{I}_{\mathrm{m}}[T]}
\end{aligned}
$$

where $T$ is the function of slip, goodness factor and motor structure, and $R_{e}$ and $I_{m}$ are the real part and image part of complex $T$, respectively.

$T$ is expressed by

$$
T=j\left[\gamma^{2}+\left(1-\gamma^{2}\right) \frac{\lambda}{a \alpha} \operatorname{th}(a \alpha)\right]
$$

where $a$ is half the primary lamination width, $\alpha$ is the ratio of $c$ to $\tau$, and $c$ is half the width of the secondary sheet overhang. $\gamma$ and $\lambda$ can be obatined by

$$
\begin{aligned}
\lambda & =\frac{1}{1+\frac{1}{\gamma} \operatorname{th}(a \alpha) \operatorname{th}[K(c-a)]} \\
\gamma^{2} & =\frac{1}{1+j S G}
\end{aligned}
$$

The five parameters in the T-circuit: primary resistance $r_{1}$, primary leakage reactance $x_{1}$, secondary resistance $r_{2}$, secondary leakage reactance $x_{2}$ and exciting reactance $x_{m}$, are derived below.

The primary resistance $r_{1}$ is

$$
r_{1}=\rho_{C u} \times 2 l_{a v} W_{1} / S_{c u}
$$

where $\rho_{C u}$ is the resistivity of copper, $l_{a v}$ is half the average length of the primary winding loop, $W_{1}$ is the number of turns of the primary winding in series, and $S_{c u}$ is the cross sectional area of the primary winding conductor.

The primary leakage reactance $X_{1}$ is

$$
X_{1}=0.158 f_{1} W_{1}^{2} \frac{a_{1}}{q}\left(\frac{\lambda_{s}}{P}+\frac{\lambda_{t}+\lambda_{e}+\lambda_{d}}{P_{e}}\right)
$$

where $f_{1}$ is the primary frequency, $a_{1}$ is the width of primary lamination, $P$ is the number of actual pole pairs, $\lambda_{\mathrm{s}}$ is the primary slot leakage magnetic conductance, $\lambda_{\mathrm{t}}$ is primary teeth leakage magnetic conductance, $\lambda_{\mathrm{e}}$ is primary winding end leakage magnetic conductance, $\lambda_{\mathrm{d}}$ is primary harmonic leakage magnetic conductance.

The secondary resistance is composed of those of conducting sheet and back iron because the flux can penetrate through the aluminum or copper sheet [8], and then enter the back iron.

The depth of flux density into back iron, $d_{F e}$, is

$$
\Delta d_{F e}=\sqrt{\frac{2 \rho_{F e}}{\omega \mu_{F e}}}
$$

where $\rho_{\mathrm{Fe}}$ is back iron resistivity, $\mu_{\mathrm{Fe}}$ is the permeability of back iron.

The resistance of secondary conducting sheet $r_{2 \text { sheet }}$ is 


$$
r_{2 \text { sheet }}=4 m_{1} \rho_{\text {second }} \frac{\left(W_{1} K_{W 1}\right)^{2}}{2 P_{e}} \frac{a_{1}}{d \tau}
$$

where $m_{1}$ is the number of primary winding phases, $\rho_{\text {second }}$ is the resistivity of the secondary electric sheet, and $K_{\mathrm{w} 1}$ is primary winding coefficient.

The resistance of secondary back iron $r_{2 \mathrm{Fe}}$ is

$$
r_{2 F e}=4 m_{1} \rho_{F e} \frac{\left(W_{1} K_{W 1}\right)^{2}}{2 P_{e}} \frac{a_{1}}{\Delta d_{F e} \tau}
$$

Therefore, the secondary equivalent resistance $r_{2}$ is

$$
r_{2}=\frac{r_{2 \text { sheet }} r_{2 F e}}{r_{2 \text { sheet }}+r_{2 F e}}
$$

The secondary leakage reactance is

$$
X_{2}=K_{f} \frac{r_{2}}{S} B_{1} \operatorname{sh}(2 K d)
$$

The exciting reaction is

$$
X_{m}=4 m_{1} \mu_{0}\left(W_{1} K_{W_{1}}\right)^{2} \frac{a_{1} V_{s}}{2 \pi \delta_{e}^{\prime} P_{e}}
$$

where $V_{\mathrm{s}}$ is the synchronous velocity of primary side.

The characteristics of SLIM are fully considered in the above circuit. When $K_{\mathrm{r}}(\mathrm{s})=K_{\mathrm{x}}(\mathrm{s})=C_{\mathrm{r}}(\mathrm{s})=C_{\mathrm{x}}(\mathrm{s})=1$, the circuit can be simplified as the same as that of RIM. Therefore, it is very convenient to analyze the performance of SLIM in the similar way as that of RIM based on the circuit [9].

\section{Optimal Design Equations Of SLiM}

As an example, this paper does optimal design on an SLIM, which was produced by a company and adopted in a linear metro but does not meet the anticipant requirement. According to their demand, a further optimization on original dimensions has been made. The efficiency and primary weight are regarded as objective functions under the given thrust as follows.

$$
\begin{cases}\min & f_{1}(x)=-\eta=-\frac{p_{2}}{p_{1}} \\ \min & f_{2}(x)=G_{\text {teeth }}+G_{\text {york }}+G_{\text {winding }}\end{cases}
$$

where $p_{1}$ is the input active power, $p_{2}$ is the output active power, $G_{\text {teeth }}$ is the primary teeth weight, $G_{\text {york }}$ is the primary yoke weight, and $G_{\text {winding }}$ is the primary winding weight.

The following parameters are selected as the constraint variables, including efficiency $(\eta)$, power factor $(\cos \varphi)$, product of power factor and efficiency $(\eta \cos \varphi)$, flux density in primary tooth $\left(B_{t}\right)$, primary length $(L)$, primary weight $\left(W_{\mathrm{p}}\right)$, thrust $\left(F_{\mathrm{e}}\right)$, vertical force $\left(F_{\mathrm{n}}\right)$, primary phase voltage $(U)$, primary phase current $(I)$, and primary conductor current density $(J)$ [10].

The other parameters are taken as design variables, such as slip $(s)$, stack height $\left(l_{\delta}\right)$, slot depth $\left(h_{\mathrm{t}}\right)$, slot width $\left(b_{\mathrm{s}}\right)$, primary height $\left(h_{\mathrm{a}}\right)$, pole pitch $(\tau)$, short pitch factor $(\beta)$, and number of turns per phase in series $\left(N_{\mathrm{s}}\right)$ [11]. The parameters involving subscript " 0 " are original ones, and the remaining are the optimal results.

The constraint conditions are given by

$$
\begin{gathered}
g_{1}(x)=\left(\eta_{0}-\eta\right) / \eta_{0} \leq 0 \\
g_{2}(x)=\left(\cos \varphi_{0}-\cos \varphi\right) / \cos \varphi_{0} \leq 0 \\
g_{3}(x)=\left(\eta_{0} \cos \varphi_{0}-\eta \cos \varphi\right) / \eta \cos \varphi \leq 0 \\
g_{4}(x)=\left(B_{t}-B_{t 0}\right) / B_{t 0} \leq 0 \\
g_{5}(x)=\left(L-L_{0}\right) / L_{0} \leq 0 \\
g_{6}(x)=\left(W_{p}-W_{p 0}\right) / W_{p 0} \leq 0 \\
g_{7}(x)=\left(F_{e 0}-F_{e}\right) / F_{e} \leq 0 \\
g_{8}(x)=\left(F_{n}-F_{n 0}\right) / F_{n 0} \leq 0 \\
g_{9}(x)=\left(U-U_{0}\right) / U_{0} \leq 0 \\
g_{10}(x)=\left(I-I_{0}\right) / I_{0} \leq 0 \\
g_{11}(x)=\left(J-J_{0}\right) / J_{0} \leq 0
\end{gathered}
$$

The above equations (20)-(31) are nonlinear functions influenced by many variables, such as structural parameters and electrical variables. They are solved by both genetic algorithm (GA) and mixture penalty function method. The GA can convert the nonlinear constraint functions into linear sub-problems so as to find a reasonable range of final results very quickly. The mixture penalty function can keep the solutions back to rationale scope by rectification when they are out of the constraint extent [11].

During the optimization process, the rated velocity is set as $36 \mathrm{~km} / \mathrm{h}$.

\section{OPTIMAL RESULTS}

TABLE I

MAIN DIMENSIONS OF SLIM

\begin{tabular}{|c|c|c|c|c|c|}
\hline $\begin{array}{c}\text { Schemes } \\
\text { Items }\end{array}$ & Original & $\begin{array}{c}\text { Optimal } \\
1\end{array}$ & $\begin{array}{c}\text { Optimal } \\
2\end{array}$ & $\begin{array}{c}\text { Optimal } \\
3\end{array}$ & $\begin{array}{c}\text { Optimal } \\
4\end{array}$ \\
\hline $\begin{array}{c}\text { Stack height } \\
\text { (mm) }\end{array}$ & 266 & 266 & 266 & 266 & 266 \\
\hline $\begin{array}{c}\text { Primary length } \\
\text { (mm) }\end{array}$ & 1950 & 2341 & 2333 & 2333 & 2459 \\
\hline $\begin{array}{c}\text { Primary depth } \\
\text { (mm) }\end{array}$ & 98.6 & 119.6 & 114.8 & 114.6 & 106.8 \\
\hline Slot width (mm) & 15.87 & 19.3 & 19 & 18.8 & 19.6 \\
\hline $\begin{array}{c}\text { Tooth width } \\
\text { (mm) }\end{array}$ & 8.4 & 10.2 & 10.4 & 10.6 & 11 \\
\hline Slot depth (mm) & 58 & 83.6 & 79.1 & 74.2 & 63.6 \\
\hline Pole pitch (mm) & 291.2 & 265.5 & 264.6 & 264.6 & 275.4 \\
\hline No. of slots & 80 & 79 & 79 & 79 & 80 \\
\hline
\end{tabular}




\begin{tabular}{|c|c|c|c|c|c|}
\hline $\begin{array}{c}\text { Mechanical } \\
\text { clearance (mm) }\end{array}$ & 12 & 12 & 12 & 12 & 12 \\
\hline $\begin{array}{c}\text { No. of } \\
\text { slots/pole/phase }\end{array}$ & 4 & 3 & 3 & 3 & 3 \\
\hline Short pitch factor & $8 / 12$ & $7 / 9$ & $7 / 9$ & $7 / 9$ & $8 / 9$ \\
\hline $\begin{array}{c}\text { Conductor area } \\
\text { (mm })^{2}\end{array}$ & 67.19 & 117.78 & 109.71 & 101.83 & 100.00 \\
\hline Number of poles & 6 & 8 & 8 & 8 & 8 \\
\hline $\begin{array}{c}\text { Secondary sheet } \\
\text { thickness (mm) }\end{array}$ & 4 & 4 & 4 & 4 & 4 \\
\hline $\begin{array}{c}\text { Secondary sheet } \\
\text { width (mm) }\end{array}$ & 354 & 354 & 354 & 354 & 354 \\
\hline $\begin{array}{c}\text { Back-iron } \\
\text { thickness (mm) }\end{array}$ & 25 & 25 & 25 & 25 & 25 \\
\hline $\begin{array}{c}\text { Base velocity } \\
\text { (Km) }\end{array}$ & 40 & 40 & 40 & 40 & 40 \\
\hline Slip frequency & 6.0 & 6.0 & 5.8 & 5.7 & 5.9 \\
\hline $\begin{array}{c}\text { Primary weight } \\
\text { (?) }\end{array}$ & 628 & 662 & 632 & 628 & 659 \\
\hline Thrust (kN) & 18.60 & 18.60 & 18.60 & 18.60 & 18.60 \\
\hline Efficiency & 0.6555 & 0.7049 & 0.7022 & 0.7069 & 0.6886 \\
\hline Power factor & 0.5055 & 0.4718 & 0.4657 & 0.4778 & 0.5188 \\
\hline
\end{tabular}

The main design parameters of SLIM are listed in Table I. Four rational optimal schemes are gained, which are compared with the original one. The thrust curve is shown in Fig. 3. The efficiency, power factor, and product of power factor and efficiency are illustrated in Figs. 4-6, respectively.

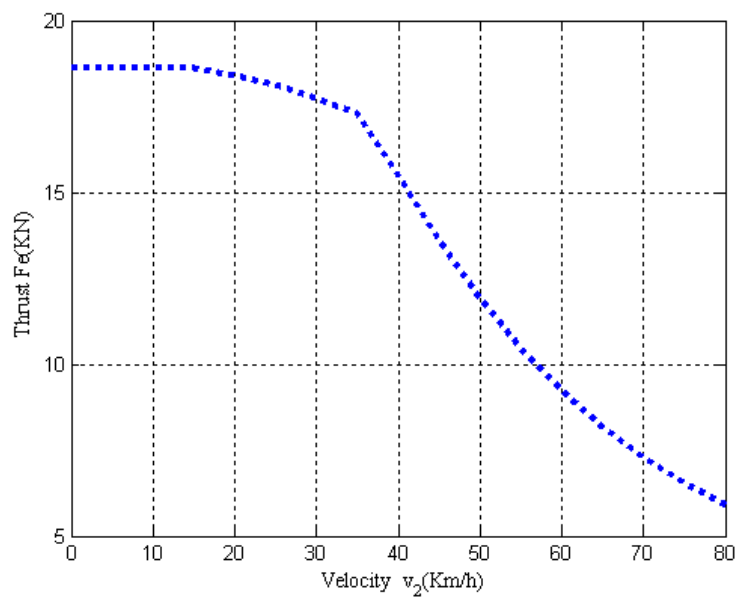

Fig.3. Curve of thrust versus velocity

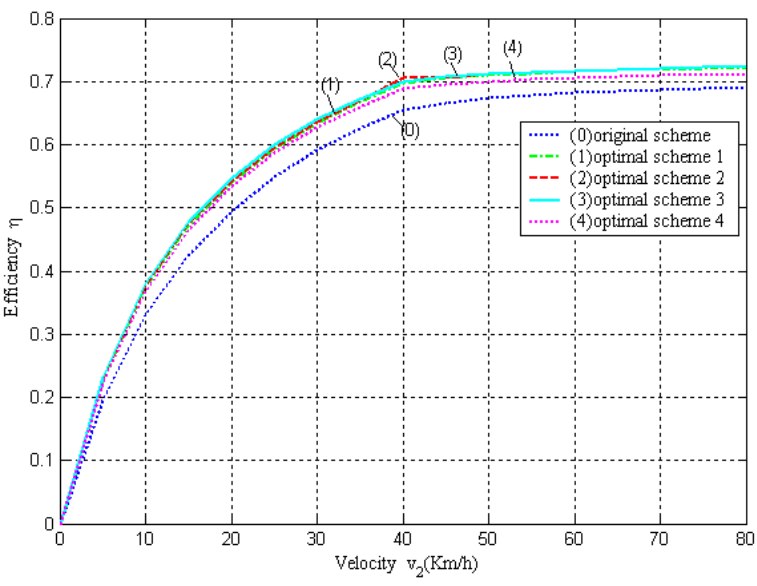

Fig. 4. Curves of efficiency versus velocity

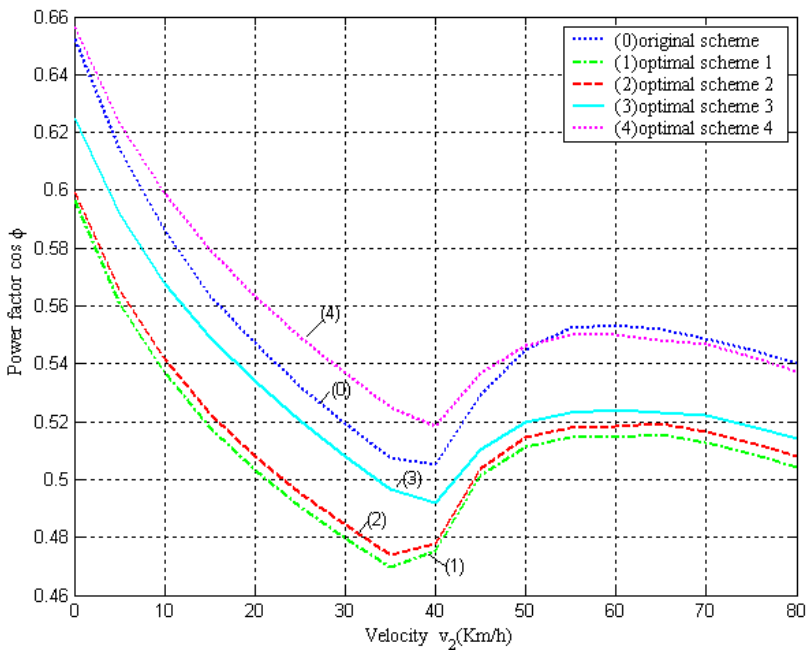

Fig. 5. Curves of power factor versus velocity

In Fig. 3, the thrust is decided by working requirement, which can overcome the wind and friction resistances, at the same time also produce definite acceleration. Below the base velocity, the thrust is kept almost constant though the phase current increases. However, beyond the base speed, the phase voltage reaches its rate value but the total reactance continues to increase, so the phase current gradually decreases as well as the thrust.

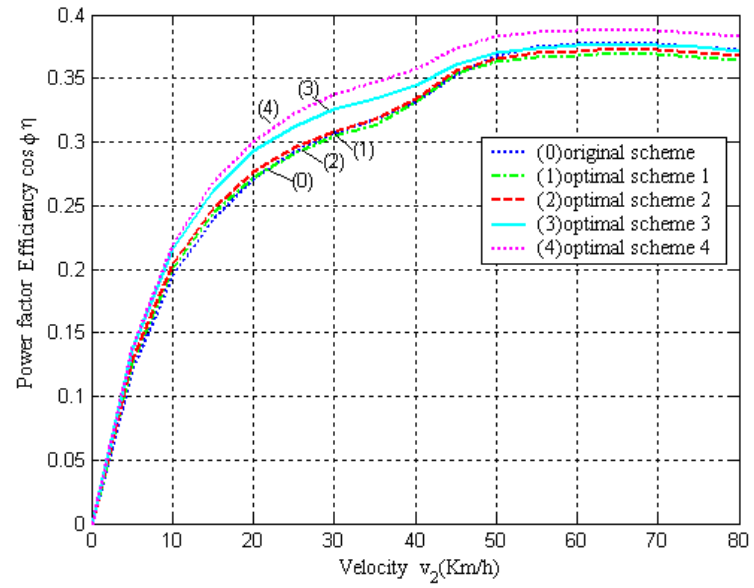


Fig. 6. Curves of product of power factor and efficiency versus velocity

From Fig. 4 to Fig. 6, we can find clearly the trend of efficiency, power factor and product of power factor and efficiency. After optimal design, the efficiency of new schemes has been improved in the whole operational range compared with the original scheme. However, the power factor in Fig. 5 encounters certain reduction in the optimal schemes. In order to attain high efficiency, the slot width, slot depth and pole pitch are changed greatly as indicated in Table I. Finally, the ratio of total resistance to reactance becomes smaller, which reduces the power factor. In Fig. 6, restrictions are made to the product of power factor and efficiency in the new schemes so as to ensure that the optimal results are not worse than the original one. In the linear drive transportation system, the inverter is placed on the vehicle. Under the same output power, the product of power factor and efficiency decides the converter rating power. That is to say, it will affect the weight of converter. Hence, it is significant to improve the efficiency while not decreasing the product of power factor efficiency. In this way, it can ensure the maximum neat load $[8,10]$.

Among the four new designs, the third is the best according to user demand. Afterwards, we make a new motor design based on the structural parameters in Table I. The performance curves are shown in Fig. 7.

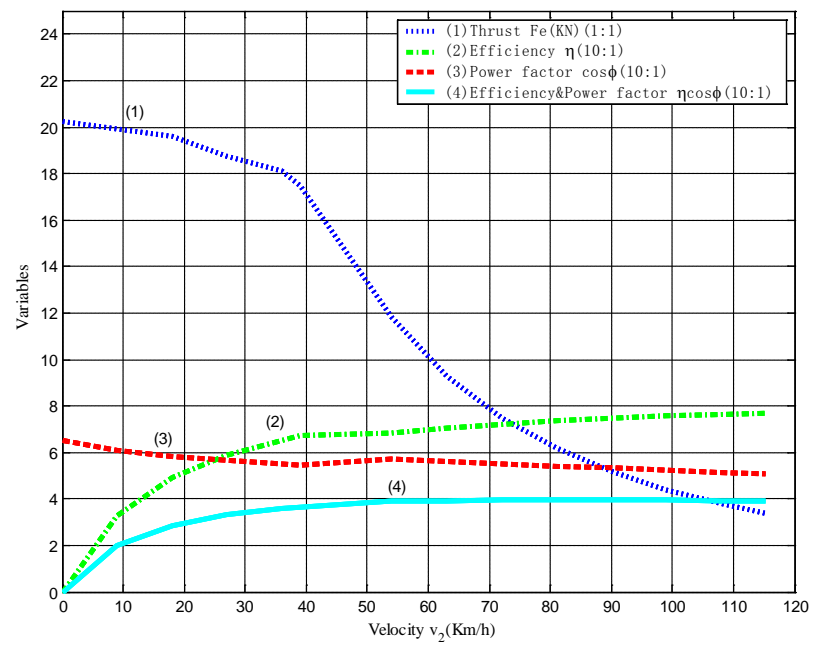

(1) First group performance curves

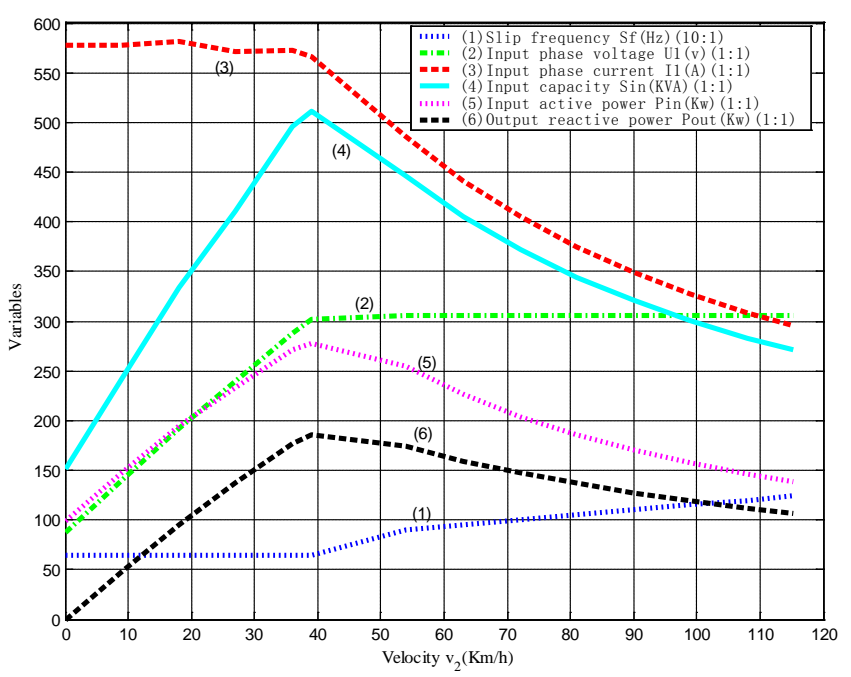

(2) Second group performance curves

Fig. 7. Optimal design performance curves

In the motor design scheme, there are two regions: "constant current" and "constant power". Below the base speed of $36 \mathrm{~km} / \mathrm{h}$, the primary phase current is kept constant (570 A), and the slip frequency is also constant $(6 \mathrm{~Hz})$. Due to power conditioner voltage limit, the constant voltage operation (with phase voltage of $306 \mathrm{~V}$ ) is applied above base speed [11].

The phase current decreases very quickly when the resistance increases. In order to meet the operating requirement, it is necessary to increase the slip frequency linearly for avoiding quick reduction of thrust. The maximum slip frequency is $12.5 \mathrm{~Hz}$ at $115 \mathrm{~km} / \mathrm{h}$.

\section{CONCLUSIONS}

In this paper, maximum efficiency optimal design of SLIM for a subway is performed. By the optimal schemes, several positive conclusions can be drawn below. Firstly, when the number of poles is larger and the pole and slip are smaller, the efficiency will be improved. Secondly, the efficiency is contradictory to power factor. It is necessary to limit the product of power factor and efficiency so as not to increase the inverter weight under the same output power. The results indicate that the equivalent circuit and optimal methods are rational.

\section{ACKNOWLEDGEMENT}

The authors are grateful to A/Prof. Yumei Du in the Institute of Electrical Engineering, Chinese Academy of Sciences, and Prof. Zhengming Zhao in the Faculty of Electrical Engineering, Tsinghua University, Beijing, China, for their helpful suggestions on the optimal schemes.

\section{REFERENCES}

[1] Wei $\mathrm{Xu}$, Yaohua $\mathrm{Li}$ and Guangsheng Sun, "Single-sided linear induction motor equivalent circuit model," The Sixth International Symposium on Linear Drives for Industrial Applications(LIDIA), Lille, France, Sept.2007. 
[2] Wei Xu, Guangshen Sun and Yaohua $\mathrm{Li}$, "Research on tractive characteristics of the single linear induction motor," IEEE Conference on Industry Technology (ICIT), Mumbai, India, Dec.2006, pp. 35-38.

[3] Kame1 Idir, Liuchen Chang and Heping Dai, "Error-based global optimization approach for electric motor design,” IEEE Transactions on Magnetics, Vol.34, No.5, pp. 2861-2864, September 1998.

[4] Wei Xu, Guangshen Sun and Yaohua Li, "A new analyse method for performance of linear induction motor," The Fourth Power Conversion Conference (PCC), Nogoya, Japan, Apr. 2007, pp. 404-409.

[5] Tsuyoshi Higuchi and Sakutaro Nonaka, "On the design of high efficiency linear induction motors for linear metro,” Electrical Engineering in Japan,Vol.137, No.2, pp. 36-43, August 2001.

[6] Sang-Baeck Yoon, Jin Hur and Dong-Seok Hyun, "A method of optimal design of single-sided linear induction motor for transit,” IEEE Transactions on Magnetics, Vol. 33, No. 5, pp. 4215-4217, September 1997.

[7] Wei Xu, Yaohua Li and Jinqi Ren, "Performance study for linear induction motor connecting winding function method with control scheme," IEEE International Conference on Industrial Technology (ICIT), Chengdu, China, Apr. 2008, pp.1 - 5.

[8] Xialing Long, Theory and magnetic design method of linear induction motor (in Chinese), China: Science Publishing Company, 2006, pp. 40-120.

[9] Wei Xu, Guangsheng Sun and Yaohua Li, "Research on performance characteristics of linear induction motor," IEEE Conference on Industrial Electronics and Applications (ICIEA), Harbin, China, May 2007, pp. 86-89.

[11] Sakutaro Nonaka and Tsuyoshi Higuchi, "Design of single-sided linear induction motors for urban transit," IEEE Transactions on Vehicular Technology, Vol. 37, No. 3, pp. 167-173, August 1988.

[12] Wei Xu, Yaohua Li, Guangsheng Sun, and Jinqi Ren, "Performance study on high power linear induction motor in transportation," International Conference on Electrical Machines and Systems (ICEMS), Seoul, Korea, Oct.2007, pp.1025-1027. 